\title{
ANALYSIS OF $\Omega_{b}^{-}(b s s)$ AND $\Omega_{c}^{0}(c s s)$ WITH QCD SUM RULES Zhi-Gang Wang 1 \\ Department of Physics, North China Electric Power University, Baoding 071003, P. R. China
}

\begin{abstract}
In this article, we calculate the masses and the pole residues of the $\frac{1}{2}^{+}$ heavy baryons $\Omega_{c}^{0}(c s s)$ and $\Omega_{b}^{-}(b s s)$ with the QCD sum rules. The numerical values $M_{\Omega_{c}^{0}}=(2.72 \pm 0.18) \mathrm{GeV}$ (or $\left.M_{\Omega_{c}^{0}}=(2.71 \pm 0.18) \mathrm{GeV}\right)$ and $M_{\Omega_{b}^{-}}=$ $(6.13 \pm 0.12) \mathrm{GeV}$ (or $M_{\Omega_{b}^{-}}=(6.18 \pm 0.13) \mathrm{GeV}$ ) are in good agreement with the experimental data.
\end{abstract}

PACS number: 14.20.Lq, 14.20.Mr

Key words: $\Omega_{c}^{0}(c s s), \Omega_{b}^{-}(b s s)$, QCD sum rules

\section{Introduction}

The charmed and bottomed baryons which contain a heavy quark and two light quarks are particularly interesting for studying dynamics of the light quarks in the presence of a heavy quark. They behave as the QCD analogue of the familiar hydrogen bounded by the electromagnetic interaction, and serve as an excellent ground for testing predictions of the constituent quark models and heavy quark symmetry [1, 2]. The $u, d$ and $s$ quarks form an $S U(3)$ flavor triplet, $\mathbf{3} \times \mathbf{3}=\overline{\mathbf{3}}+\mathbf{6}$, two light quarks can form diquarks of a symmetric sextet and an antisymmetric antitriplet. For the $S$-wave baryons, the sextet contains both spin- $\frac{1}{2}$ and spin- $\frac{3}{2}$ states, while the antitriplet contains only spin- $\frac{1}{2}$ states.

There have been many theoretical approaches dealing with the heavy baryons with one heavy quark, for example, the relativized potential quark model [3], the Feynman-Hellmann theorem and semi-empirical mass formulas [4, the Faddeev equation [5, 6], the combined expansion in $1 / m_{Q}$ and $1 / N_{c}$ [7], the lattice simulations [8, 9], the relativistic quark-diquark approximation [10, 11, the hyperfine interactions [12], the QCD sum rules in the heavy quark effective field theory [13], the variational approach [14, and the full QCD sum rules [15, 16, etc.

The $\frac{1}{2}^{+}$antitriplet states $\left(\Lambda_{c}^{+}, \Xi_{c}^{+}, \Xi_{c}^{0}\right)$, and the $\frac{1}{2}^{+}$and $\frac{3}{2}^{+}$sextet states $\left(\Omega_{c}, \Sigma_{c}, \Xi_{c}^{\prime}\right)$ and $\left(\Omega_{c}^{*}, \Sigma_{c}^{*}, \Xi_{c}^{*}\right)$ have been established; while the corresponding bottomed baryons are far from complete, only the $\Lambda_{b}, \Sigma_{b}, \Sigma_{b}^{*}, \Xi_{b}$ have been observed [17].

Recently the D0 collaboration reported the observation of the doubly strange baryon $\Omega_{b}^{-}$in the decay channel $\Omega_{b}^{-} \rightarrow J / \psi \Omega^{-}$(with $J / \psi \rightarrow \mu^{+} \mu^{-}$and $\Omega^{-} \rightarrow$ $\left.\Lambda K^{-} \rightarrow p \pi^{-} K^{-}\right)$in $p \bar{p}$ collisions at $\sqrt{s}=1.96 \mathrm{TeV}$ [18]. The experimental value $M_{\Omega_{b}^{-}}=6.165 \pm 0.010 \pm 0.013 \mathrm{GeV}$ is about $0.1 \mathrm{GeV}$ larger than the existing theoretical calculations [4, 6, 7, 8, 9, 10, 11, 12, 13, 14, while the theoretical prediction

\footnotetext{
${ }^{1}$ E-mail,wangzgyiti@yahoo.com.cn.
} 
$M_{\Omega_{c}^{0}} \approx 2.7 \mathrm{GeV}$ [4, 6, 7, 8, 9, 10, 11, 12, 13, 14, is consistent with the experimental data $M_{\Omega_{c}^{0}}=(2.6975 \pm 0.0026) \mathrm{GeV}$ [17] .

In previous work, we have calculated the masses and the pole residues of the $\frac{3}{2}^{+}$ heavy baryons $\Omega_{c}^{*}$ and $\Omega_{b}^{*}$ with the QCD sum rules [19]. In this article, we extend our previous work to study the corresponding $\frac{1}{2}^{+}$heavy baryons $\Omega_{c}^{0}$ and $\Omega_{b}^{-}$.

In Ref. [20], the masses of the heavy baryons $\Omega_{c}^{0}$ and $\Omega_{b}^{-}$are studied in the full QCD sum rules with the same interpolating current as the present work. The authors choose the tensor structure $\not p$ and obtain different spectral density 2 .

The masses of the $\Lambda_{Q}, \Sigma_{Q}$ and $\Xi_{Q}$ have been calculated with the full QCD sum rules [15, 16, 20]. The masses of the $\Sigma_{Q}^{*}, \Sigma_{Q}$ and $\Lambda_{Q}$ have been calculated with the QCD sum rules in the leading order of the heavy quark effective theory [21, 22, 23], and later the $1 / m_{Q}$ corrections were studied [24, 25, 26]. Furthermore, the masses of the orbitally excited heavy baryons with the leading order approximation [27, 28] and the $1 / m_{Q}$ corrections [29] in the heavy quark effective theory have also been analyzed. Recently the $\frac{1}{2}^{+}$and $\frac{3}{2}^{+}$bottomed baryons were studied with the QCD sum rules in the heavy quark effective theory including the $1 / m_{Q}$ corrections [13].

In the QCD sum rules, the operator product expansion is used to expand the time-ordered currents into a series of quark and gluon condensates which parameterize the long distance properties of the QCD vacuum. Based on the quark-hadron duality, we can obtain copious information about the hadronic parameters at the phenomenological side [30, 31].

The article is arranged as follows: we derive the QCD sum rules for the masses and the pole residues of the $\Omega_{c}^{0}$ and $\Omega_{b}^{-}$in section 2 ; in section 3 numerical results are given and discussed, and section 4 is reserved for conclusion.

\section{QCD sum rules for the $\Omega_{c}^{0}$ and $\Omega_{b}^{-}$}

In the following, we write down the two-point correlation functions $\Pi^{a}(p)$ in the QCD sum rules approach,

$$
\begin{aligned}
\Pi^{a}(p) & =i \int d^{4} x e^{i p \cdot x}\left\langle 0\left|T\left\{J^{a}(x) \bar{J}^{a}(0)\right\}\right| 0\right\rangle, \\
J^{a}(x) & =\epsilon^{i j k} s_{i}^{T}(x) C \gamma_{\mu} s_{j}(x) \gamma_{5} \gamma^{\mu} Q_{k}^{a}(x), \\
\lambda_{a} N(p, s) & =\left\langle 0\left|J^{a}(0)\right| \Omega_{a}(p, s)\right\rangle,
\end{aligned}
$$

where the upper index $a$ represents the $c$ and $b$ quarks respectively, $i, j$ and $k$ are color indexes, $C$ is the charge conjunction matrix, the $N(p, s)$ and $\lambda_{a}$ stand for the Dirac spin vector and the pole residue of the heavy baryon $\Omega_{a}$, respectively.

\footnotetext{
${ }^{2}$ When I finish the work and submit it to the network http://arXiv.org, I learn from Prof. Nielsen that they have studied the masses of the heavy baryons $\Omega_{c}^{0}$ and $\Omega_{b}^{-}$in the full QCD sum rules with the same interpolating current $J^{a}(x)$ [20].
} 
The correlation functions $\Pi^{a}(p)$ can be decomposed as follows:

$$
\Pi^{a}(p)=\not p \Pi_{1}^{a}(p)+\Pi_{2}^{a}(p)
$$

due to Lorentz covariance. The first structure $\not p$ has an odd number of $\gamma$-matrices and conserves chirality, the second structure 1 has an even number of $\gamma$-matrices and violate chirality. In the original QCD sum rules analysis of the nucleon masses and magnetic moments [32, 33, 34, 35], the interval of dimensions (of the condensates) for the odd structure is larger than the interval of dimensions for the even structure, one may expect a better accuracy of the results obtained from the sum rules with the odd structure.

In this article, we choose the two tensor structures to study the masses and the pole residues of the heavy baryons $\Omega_{c}^{0}$ and $\Omega_{b}^{-}$, as the masses of the heavy quarks break the chiral symmetry explicitly.

The components $\Pi_{1}^{a}(p)$ with the odd structure have smaller dimension of mass than the components $\Pi_{2}^{a}(p)$ with the even structure, naively we expect the components $\Pi_{1}^{a}(p)$ have better convergent behavior in the operator product expansion, and the numerical results confirm this conjecture, see Tables 1-2.

We insert a complete set of intermediate baryon states with the same quantum numbers as the current operators $J^{a}(x)$ into the correlation functions $\Pi^{a}(p)$ to obtain the hadronic representation [30, 31]. After isolating the pole terms of the lowest states $\Omega_{a}$, we obtain the following result:

$$
\Pi^{a}(p)=\lambda_{a}^{2} \frac{M_{\Omega_{a}}+\not p}{M_{\Omega_{a}}^{2}-p^{2}}+\cdots .
$$

In the following, we briefly outline the operator product expansion for the correlation functions $\Pi^{a}(p)$ in perturbative QCD. The calculations are performed at large space-like momentum region $p^{2} \ll 0$, which corresponds to small distance $x \approx 0$ required by validity of the operator product expansion. We write down the "full" propagators $S_{i j}(x)$ and $S_{Q}^{i j}(x)$ of a massive quark in the presence of the vacuum condensates firstly [31],

$$
\begin{aligned}
S_{i j}(x)= & \frac{i \delta_{i j} \not x}{2 \pi^{2} x^{4}}-\frac{\delta_{i j} m_{s}}{4 \pi^{2} x^{2}}-\frac{\delta_{i j}}{12}\langle\bar{s} s\rangle+\frac{i \delta_{i j}}{48} m_{s}\langle\bar{s} s\rangle \not x-\frac{\delta_{i j} x^{2}}{192}\left\langle\bar{s} g_{s} \sigma G s\right\rangle \\
& +\frac{i \delta_{i j} x^{2}}{1152} m_{s}\left\langle\bar{s} g_{s} \sigma G s\right\rangle \not x-\frac{i}{32 \pi^{2} x^{2}} G_{\mu \nu}^{i j}\left(\not x \sigma^{\mu \nu}+\sigma^{\mu \nu} \not x\right)+\cdots, \\
S_{Q}^{i j}(x)= & \frac{i}{(2 \pi)^{4}} \int d^{4} k e^{-i k \cdot x}\left\{\frac{\delta_{i j}}{\not k-m_{Q}}-\frac{g_{s} G_{i j}^{\alpha \beta}}{4} \frac{\sigma_{\alpha \beta}\left(\not k+m_{Q}\right)+\left(\not k+m_{Q}\right) \sigma_{\alpha \beta}}{\left(k^{2}-m_{Q}^{2}\right)^{2}}\right. \\
& \left.+\frac{\pi^{2}}{3}\left\langle\frac{\alpha_{s} G G}{\pi}\right\rangle \delta_{i j} m_{Q} \frac{k^{2}+m_{Q} \not k}{\left(k^{2}-m_{Q}^{2}\right)^{4}}+\cdots\right\},
\end{aligned}
$$

where $\left\langle\bar{s} g_{s} \sigma G s\right\rangle=\left\langle\bar{s} g_{s} \sigma_{\alpha \beta} G^{\alpha \beta} s\right\rangle$ and $\left\langle\frac{\alpha_{s} G G}{\pi}\right\rangle=\left\langle\frac{\alpha_{s} G_{\alpha \beta} G^{\alpha \beta}}{\pi}\right\rangle$, then contract the quark 
fields in the correlation functions $\Pi^{a}(p)$ with Wick theorem, and obtain the result:

$$
\Pi^{a}(p)=-2 i \epsilon^{i j k} \epsilon^{i^{\prime} j^{\prime} k^{\prime}} \int d^{4} x e^{i p \cdot x} \operatorname{Tr}\left[\gamma_{\mu} S_{i i^{\prime}}(x) \gamma_{\nu} C S_{j j^{\prime}}^{T}(x) C\right] \gamma_{5} \gamma_{\mu} S_{Q}^{k k^{\prime}}(x) \gamma_{\nu} \gamma_{5}
$$

Substitute the full $s, c$ and $b$ quark propagators into above correlation functions and complete the integral in the coordinate space, then integrate over the variable $k$, we can obtain the correlation functions $\Pi_{i}^{a}(p)$ at the level of quark-gluon degree of freedom. Once analytical results are obtained, then we can take the quark-hadron duality and perform Borel transform with respect to the variable $P^{2}=-p^{2}$, finally we obtain the following two sum rules with respect to the tensor structures $\not p$ and 1 respectively:

$$
\begin{aligned}
& \lambda_{a}^{2} \exp \left(-\frac{M_{\Omega_{a}}^{2}}{M^{2}}\right)=\frac{1}{16 \pi^{4}} \int_{t h}^{s_{a}^{0}} d s \int_{\Delta^{a}}^{1} d x x(1-x)^{3}\left(\widetilde{m}_{a}^{2}-s\right)\left(3 \widetilde{m}_{a}^{2}-5 s\right) \exp \left(-\frac{s}{M^{2}}\right) \\
& +\frac{m_{s}\langle\bar{s} s\rangle}{\pi^{2}} \int_{t h}^{s_{a}^{0}} d s \int_{\Delta^{a}}^{1} d x x(2-3 x) \exp \left(-\frac{s}{M^{2}}\right) \\
& +\frac{1}{48 \pi^{2}}\left\langle\frac{\alpha_{s} G G}{\pi}\right\rangle \int_{t h}^{s_{a}^{0}} d s \int_{\Delta^{a}}^{1} d x(4-5 x) \exp \left(-\frac{s}{M^{2}}\right) \\
& +\frac{m_{s}\langle\bar{s} s\rangle}{\pi^{2}} \int_{0}^{1} d x x(1-x) \widetilde{m}_{a}^{2} \exp \left(-\frac{\widetilde{m}_{a}^{2}}{M^{2}}\right) \\
& -\frac{m_{s}\left\langle\bar{s} g_{s} \sigma G s\right\rangle}{6 \pi^{2}} \int_{0}^{1} d x x\left(2+\frac{\widetilde{m}_{a}^{2}}{M^{2}}\right) \exp \left(-\frac{\tilde{m}_{a}^{2}}{M^{2}}\right) \\
& +\frac{1}{144 \pi^{2}}\left\langle\frac{\alpha_{s} G G}{\pi}\right\rangle \int_{0}^{1} d x\left(\frac{2 x^{3}-9 x^{2}+9 x-2}{x}-\frac{(1-x)^{3} \widetilde{m}_{a}^{2}}{x M^{2}}\right) \\
& \widetilde{m}_{a}^{2} \exp \left(-\frac{\widetilde{m}_{a}^{2}}{M^{2}}\right) \\
& +\frac{2\langle\bar{s} s\rangle^{2}}{3} \exp \left(-\frac{m_{a}^{2}}{M^{2}}\right)+\frac{m_{s}\left\langle\bar{s} g_{s} \sigma G s\right\rangle}{4 \pi^{2}} \exp \left(-\frac{m_{a}^{2}}{M^{2}}\right) \\
& M_{\Omega_{a}} \lambda_{a}^{2} \exp \left(-\frac{M_{\Omega_{a}}^{2}}{M^{2}}\right)=\frac{3 m_{a}}{32 \pi^{4}} \int_{t h}^{s_{a}^{0}} d s \int_{\Delta^{a}}^{1} d x(1-x)^{2}\left(\widetilde{m}_{a}^{2}-s\right)^{2} \exp \left(-\frac{s}{M^{2}}\right) \\
& -\frac{3 m_{a} m_{s}\langle\bar{s} s\rangle}{2 \pi^{2}} \int_{t h}^{s_{a}^{0}} d s \int_{\Delta^{a}}^{1} d x \exp \left(-\frac{s}{M^{2}}\right) \\
& +\frac{m_{a}}{96 \pi^{2}}\left\langle\frac{\alpha_{s} G G}{\pi}\right\rangle \int_{t h}^{s_{a}^{0}} d s \int_{\Delta^{a}}^{1} d x\left(\frac{2}{x^{2}}-2 x-3\right) \exp \left(-\frac{s}{M^{2}}\right) \\
& -\frac{m_{a}}{96 \pi^{2}}\left\langle\frac{\alpha_{s} G G}{\pi}\right\rangle \int_{0}^{1} d x \frac{(1-x)^{2}}{x} \widetilde{m}_{a}^{2} \exp \left(-\frac{\widetilde{m}_{a}^{2}}{M^{2}}\right) \\
& +\frac{5 m_{a} m_{s}\left\langle\bar{s} g_{s} \sigma G s\right\rangle}{12 \pi^{2}} \exp \left(-\frac{m_{a}^{2}}{M^{2}}\right)+\frac{4 m_{a}\langle\bar{s} s\rangle^{2}}{3} \exp \left(-\frac{m_{a}^{2}}{M^{2}}\right) \text {, }
\end{aligned}
$$


where $t h=\left(m_{a}+2 m_{s}\right)^{2}, \Delta^{a}=\frac{m_{a}^{2}}{s}$ and $\widetilde{m}_{a}^{2}=\frac{m_{a}^{2}}{x}$.

We carry out the operator product expansion to the vacuum condensates adding up to dimension- 6 and calculate the same Feynman diagrams as in the sum rules for the $\frac{3}{2}^{+}$heavy baryons [19] (where the two tensor structures $g_{\mu \nu} \not p$ and $g_{\mu \nu}$ are chosen), the contribution from each of the Feynman diagrams differs from the corresponding one in our previous work.

In calculation, we take assumption of vacuum saturation for the high dimension vacuum condensates, they are always factorized to lower condensates with vacuum saturation in the QCD sum rules, factorization works well in large $N_{c}$ limit. In this article, we take into account the contributions from the quark condensates $\langle\bar{s} s\rangle,\langle\bar{s} s\rangle^{2}$, mixed condensate $\left\langle\bar{s} g_{s} \sigma G s\right\rangle$, gluon condensate $\left\langle\frac{\alpha_{s} G G}{\pi}\right\rangle$, and neglect the contributions from other high dimension condensates, which are suppressed by large denominators and would not play significant roles.

Differentiate the above sum rules with respect to the variable $\frac{1}{M^{2}}$, then eliminate the pole residue $\lambda_{\Omega_{a}}$, we obtain two QCD sum rules for the masses $M_{\Omega_{a}}$ with respect to the tensor structures $\not p$ and 1 , respectively.

In the heavy quark limit $m_{Q} \rightarrow \infty$, the quarks $c$ and $b$ degenerate, we replace the heavy quark field $Q_{a}(x)$ in the baryon current $J^{a}(x)$ with the effective field $h(x)$ to obtain the interpolating current $J_{h}(x)=\epsilon^{i j k} s_{i}^{T}(x) C \gamma_{\mu} s_{j}(x) \gamma_{5} \gamma^{\mu} h_{k}(x)$ in the heavy quark effective theory, which differs from the corresponding one constructed from the heavy quark effective theory remarkably and does not warrant the tensor structure $\frac{1+\mathrm{te} y}{2}[22]$.

In the heavy quark limit, the correlation function $\Pi^{a}(\omega)$ (with $\omega=v \cdot p$ ) can be written as $\Pi^{a}(\omega)=\not \Pi_{1}(\omega)+\Pi_{2}(\omega)\left(\Pi_{1}(\omega) \neq \Pi_{2}(\omega)\right)$. In Ref. [13], the current operator $\eta(x)=\epsilon^{i j k} s_{i}^{T}(x) C \gamma_{\mu} s_{j}(x) \gamma_{5}\left[\gamma^{\mu}-\not v^{\mu}\right] h_{k}(x)$ is used to interpolate the baryons $\Omega_{a}$, and the correlation function is written as $\Pi^{a}(\omega)=\frac{1+0 /}{2} \bar{\Pi}(\omega)$.

The effective heavy quark propagator has the following form,

$$
S_{h}^{a b}(x)=\delta_{a b} \frac{1+\not p}{2} \int_{0}^{\infty} d t \delta(x-v t),
$$

where the $v_{\mu}$ is a four-vector with $v^{2}=1$. The calculations of the operator product expansion can be performed in the coordinate space and are greatly facilitated, as we do not need the mixed picture both in coordinate and momentum spaces. We calculate the same Feynman diagrams as in the full QCD, the contributions of some diagrams vanish in the heavy quark limit.

Finally we obtain two sum rules with respect to the tensor structures $\not p$ and 1 
respectively:

$$
\begin{aligned}
F^{2} \exp \left(-\frac{\bar{\Lambda}}{T}\right)= & \frac{1}{5 \pi^{4}} \int_{2 m_{s}}^{\omega_{0}} d \omega \omega^{5} \exp \left(-\frac{\omega}{T}\right)+\frac{m_{s}\left\langle\bar{s} g_{s} \sigma G s\right\rangle}{24 \pi^{2}}+\frac{\langle\bar{s} s\rangle^{2}}{3} \\
F^{2} \exp \left(-\frac{\bar{\Lambda}}{T}\right)= & \frac{1}{10 \pi^{4}} \int_{2 m_{s}}^{\omega_{0}} d \omega \omega^{5} \exp \left(-\frac{\omega}{T}\right)-\frac{3 m_{s}\langle\bar{s} s\rangle}{\pi^{2}} \int_{2 m_{s}}^{\omega_{0}} d \omega \omega \exp \left(-\frac{\omega}{T}\right) \\
& -\frac{1}{16 \pi^{2}}\left\langle\frac{\alpha_{s} G G}{\pi}\right\rangle \int_{2 m_{s}}^{\omega_{0}} d \omega \omega \exp \left(-\frac{\omega}{T}\right)+\frac{5 m_{s}\left\langle\bar{s} g_{s} \sigma G s\right\rangle}{24 \pi^{2}}+\frac{2\langle\bar{s} s\rangle^{2}}{3}
\end{aligned}
$$

where we have used the definitions for the binding energy $\bar{\Lambda}$ and the pole residue $F$, limit $_{m_{Q} \rightarrow \infty} M_{\Omega}=m_{Q}+\bar{\Lambda}$ and $\langle 0|J(0)| \Omega(v, s)\rangle=F N(v, s)$, the $N(v, s)$ is the Dirac spin vector in the heavy quark limit.

Differentiate the above sum rules with respect to the variable $\frac{1}{T}$, then eliminate the pole residue $F$, we obtain two QCD sum rules for the binding energy $\bar{\Lambda}$ with respect to the tensor structures $\not 6$ and 1 , respectively.

\section{$3 \quad$ Numerical results and discussions}

The input parameters are taken to be the standard values $\langle\bar{q} q\rangle=-(0.24 \pm 0.01 \mathrm{GeV})^{3}$, $\langle\bar{s} s\rangle=(0.8 \pm 0.2)\langle\bar{q} q\rangle,\left\langle\bar{s} g_{s} \sigma G s\right\rangle=m_{0}^{2}\langle\bar{s} s\rangle, m_{0}^{2}=(0.8 \pm 0.2) \mathrm{GeV}^{2},\left\langle\frac{\alpha_{s} G G}{\pi}\right\rangle=$ $(0.33 \mathrm{GeV})^{4}, m_{s}=(0.14 \pm 0.01) \mathrm{GeV}, m_{c}=(1.4 \pm 0.1) \mathrm{GeV}$ and $m_{b}=(4.8 \pm 0.1) \mathrm{GeV}$ at the energy scale about $\mu=1 \mathrm{GeV}$ [30, 31, 36]. The contribution from the gluon condensate $\left\langle\frac{\alpha_{s} G G}{\pi}\right\rangle$ is less than $3 \%$, and the uncertainty is neglected here.

We usually consult the experimental data in choosing the Borel parameter $M^{2}$ and the threshold parameter $s_{0}$. There lack experimental data for the phenomenological hadronic spectral densities of the bottomed baryons at present, only the $\Lambda_{b}$, $\Sigma_{b}, \Sigma_{b}^{*}, \Xi_{b}$ [17] and $\Omega_{b}^{-}$[18] have been observed, we can borrow some ideas from the light baryon spectra [17].

For the octet baryons with $J^{P}=\frac{1}{2}^{+}$, the mass of the proton is $M_{p}=938 \mathrm{MeV}$, and the mass of the first radial excited state $N(1440)$ (the Roper resonance) is $M_{1440}=(1420-1470) \mathrm{MeV} \approx 1440 \mathrm{MeV}$; the mass of the ground state $\Sigma$ is $M_{\Sigma}=$ $1189.37 \mathrm{MeV}$, and the mass of the first radial excited state $\Sigma(1660)$ is $M_{1660}=$ $(1630-1690) \mathrm{MeV} \approx 1660 \mathrm{MeV}$; the mass of the ground state $\Xi$ is $M_{\Xi}=1321.7 \mathrm{MeV}$, while the spin-parity of the high excited states $\Xi(1620), \Xi(1690), \Xi(1950), \Xi(2030)$, $\Xi(2120), \Xi(2250), \Xi(2370), \Xi(2500)$ have not been determined yet [17. For the decuplet baryons with $J^{P}=\frac{3}{2}^{+}$, the mass of the $\Delta(1232)$ is $M_{1232}=(1231-$ $1233) \mathrm{MeV} \approx 1232 \mathrm{MeV}$, and the mass of the first radial excited state $\Delta(1600)$ is $M_{1600}=(1550-1700) \mathrm{MeV} \approx 1600 \mathrm{MeV}$; the mass of the ground state $\Sigma(1385)$ is $M_{1385}=1382.8 \mathrm{MeV}$, and the mass of the first radial excited state $\Sigma(1840)$ is $M_{1840}=1840 \mathrm{MeV}$; the ground states $\Xi(1530), \Omega(1672)$ are well established, while the spin-parity of the high excited states $\Omega(2250), \Omega(2380), \Omega(2470)$ have not been determined yet [17]. 


\begin{tabular}{|c|c|c|}
\hline \hline & Eq.(8) & Eq.(9) \\
\hline perturbative term & $+93 \%$ & $+70 \%$ \\
\hline$\langle\bar{s} s\rangle$ & $-1 \%$ & $+21 \%$ \\
\hline$\left\langle\bar{s} g_{s} \sigma G s\right\rangle$ & $-1 \%$ & $-3 \%$ \\
\hline$\langle\bar{s} s\rangle^{2}$ & $+8 \%$ & $+10 \%$ \\
\hline$\left\langle\frac{\alpha_{s} G G}{\pi}\right\rangle$ & $+1 \%$ & $+1 \%$ \\
\hline \hline
\end{tabular}

Table 1: The contributions from different terms in the sum rules for the $\Omega_{c}^{0}$ with the central values of the input parameters "Set I".

\begin{tabular}{|c|c|c|}
\hline \hline & Eq. (8) & Eq.(9) \\
\hline perturbative term & $+94 \%$ & $+76 \%$ \\
\hline$\langle\bar{s} s\rangle$ & $-2 \%$ & $+23 \%$ \\
\hline$\left\langle\bar{s} g_{s} \sigma G s\right\rangle$ & $-1 \%$ & $-2 \%$ \\
\hline$\langle\bar{s} s\rangle^{2}$ & $+8 \%$ & $+5 \%$ \\
\hline$\left\langle\frac{\alpha_{s} G G}{\pi}\right\rangle$ & $+1 \%$ & $-3 \%$ \\
\hline \hline
\end{tabular}

Table 2: The contributions from different terms in the sum rules for the $\Omega_{b}^{-}$with the central values of the input parameters "Set I".

From the experimental data for the baryons consist of $q q q$ and $q q s$, we can see that the energy gap between the ground states and the first radial excited states is about $(0.4-0.5) \mathrm{GeV}$, the $S U(3)$ breaking effects are rather small. So in the QCD sum rules for the baryons with the light quarks, the threshold parameters $s_{0}$ are always chosen to be $\sqrt{s_{0}}=M_{g r}+0.5 \mathrm{GeV}$ [32, 33, 34, 35], here $g r$ stands for the ground states. The central values of the threshold parameters for the heavy baryons $\Omega_{c}^{0}$ and $\Omega_{b}^{-}$can be chosen as $s_{\Omega_{c}}^{0}=(2.6975+0.5)^{2} \mathrm{GeV}^{2}$ and $s_{\Omega_{b}}^{0}=(6.165+0.5)^{2} \mathrm{GeV}^{2}$, respectively.

In this article, the threshold parameters and the Borel parameters are taken as $s_{\Omega_{c}}^{0}=(10.5 \pm 1.0) \mathrm{GeV}^{2}$ and $M^{2}=(2.2-3.2) \mathrm{GeV}^{2}$ for the charmed baryon $\Omega_{c}^{0}$, and $s_{\Omega_{b}}^{0}=(44.5 \pm 1.0) \mathrm{GeV}^{2}$ and $M^{2}=(5.0-6.0) \mathrm{GeV}^{2}$ for the bottomed baryon $\Omega_{b}^{-}$; thereafter these parameters will be denoted as "Set I". In the heavy quark limit $m_{Q} \rightarrow \infty$, the corresponding Borel parameter $T$ and threshold parameter $\omega_{0}$ can be taken as $T=(0.4-0.6) \mathrm{GeV}$ and $\omega_{0}=(1.7-1.9) \mathrm{GeV}$; and we will refer them as "Set III".

We can make another estimation for the threshold parameters by adding the heavy quark masses to the ground state $\frac{1}{2}^{+}$baryons which consist of qss, $s_{\Omega_{c}}^{0}=$ $\left(M_{\Xi}+m_{c}+0.5 \mathrm{GeV}\right)^{2} \approx 10.5 \mathrm{GeV}^{2}$ and $s_{\Omega_{b}}^{0}=\left(M_{\Xi}+m_{b}+0.5 \mathrm{GeV}\right)^{2} \approx 44.0 \mathrm{GeV}^{2}$, which are almost the same values as "Set I".

The contributions from different terms at the central values of the input parameters are presented in Table.1 and Table.2, respectively. From the two tables, we can 


\begin{tabular}{|c|c|c|}
\hline \hline & $\Omega_{c}^{0}$ & $\Omega_{b}^{-}$ \\
\hline$\not p \&$ Set I & $(34-60) \%$ & $(35-49) \%$ \\
\hline 1 \& Set I & $(34-62) \%$ & $(36-51) \%$ \\
\hline$\not p$ \& Set II & $(52-75) \%$ & $(51-66) \%$ \\
\hline 1 \& Set II & $(53-78) \%$ & $(51-67) \%$ \\
\hline \hline
\end{tabular}

Table 3: The contributions of the pole terms from different sum rules, here we take the central values of the input parameters except for the Borel parameter $M^{2}$.

expect convergence of the operator product expansion. The components $\Pi_{1}^{a}(p)$ with the odd structure have smaller dimension of mass than the components $\Pi_{2}^{a}(p)$ with the even structure, see Eq.(4), it is not unexpected that the components $\Pi_{1}^{a}(p)$ have better convergent behavior in the operator product expansion and result in better QCD sum rules, see Table 4.

In the heavy quark limit, the contribution from the quark condensate $\langle\bar{s} s\rangle$ vanishes for the odd structure $\not p$, while the condensate $\langle\bar{s} s\rangle$ has a numerical coefficient $\frac{3}{\pi^{2}}$ for the even structure 1, see Eqs.(11-12), which can explain the hierarchy appears in the operator product expansion naturally, see Tables 1-2.

The contributions of the pole terms for different sum rules are shown in Table 3 , from the table, we can see that the pole dominance condition is marginally satisfied for the parameters "Set I".

We can choose smaller Borel parameters $M^{2}$ or larger threshold parameters $s_{a}^{0}$ to enhance the contributions from the ground states. However, if we take larger threshold parameter $s_{a}^{0}$, the contribution from the first radial excited state maybe included in; on the other hand, for smaller Borel parameter $M^{2}$, the sum rules are not stable enough, the uncertainty with variation of the Borel parameter is large.

In this article, we also present the results with smaller Borel parameters $M^{2}$ and larger threshold parameters $s_{a}^{0}, s_{\Omega_{c}}^{0}=(11.0 \pm 1.0) \mathrm{GeV}^{2}$ and $M^{2}=(1.9-2.6) \mathrm{GeV}^{2}$ for the charmed baryon $\Omega_{c}^{0}$, and $s_{\Omega_{b}}^{0}=(46.5 \pm 1.0) \mathrm{GeV}^{2}$ and $M^{2}=(4.5-5.4) \mathrm{GeV}^{2}$ for the bottomed baryon $\Omega_{b}^{-}$. These parameters (denoted as "Set II") satisfy both pole dominance (see Table 3 ) and convergence of the operator product expansion.

Taking into account all uncertainties of the input parameters, finally we obtain the values of the masses and the pole residues of the heavy baryons $\Omega_{c}^{0}$ and $\Omega_{b}^{-}$, which are shown in Figs.1-4 (and Table 4), respectively.

In the heavy quark limit, we obtain the binding energy $\bar{\Lambda}$ (see Fig.5)

$$
\begin{aligned}
& \bar{\Lambda}=1.30 \pm 0.17 \mathrm{GeV}, \\
& \bar{\Lambda}=1.10 \pm 0.26 \mathrm{GeV},
\end{aligned}
$$

for the tensor structures $\not \varnothing$ and 1 respectively. The corresponding masses are presented in Table 4.

The values $M_{\Omega_{c}^{0}}=(2.72 \pm 0.18) \mathrm{GeV}$ and $M_{\Omega_{c}^{0}}=(2.71 \pm 0.18) \mathrm{GeV}$ from the sum rules with the tensor structure $\not p$ are in good agreement with the experimental 

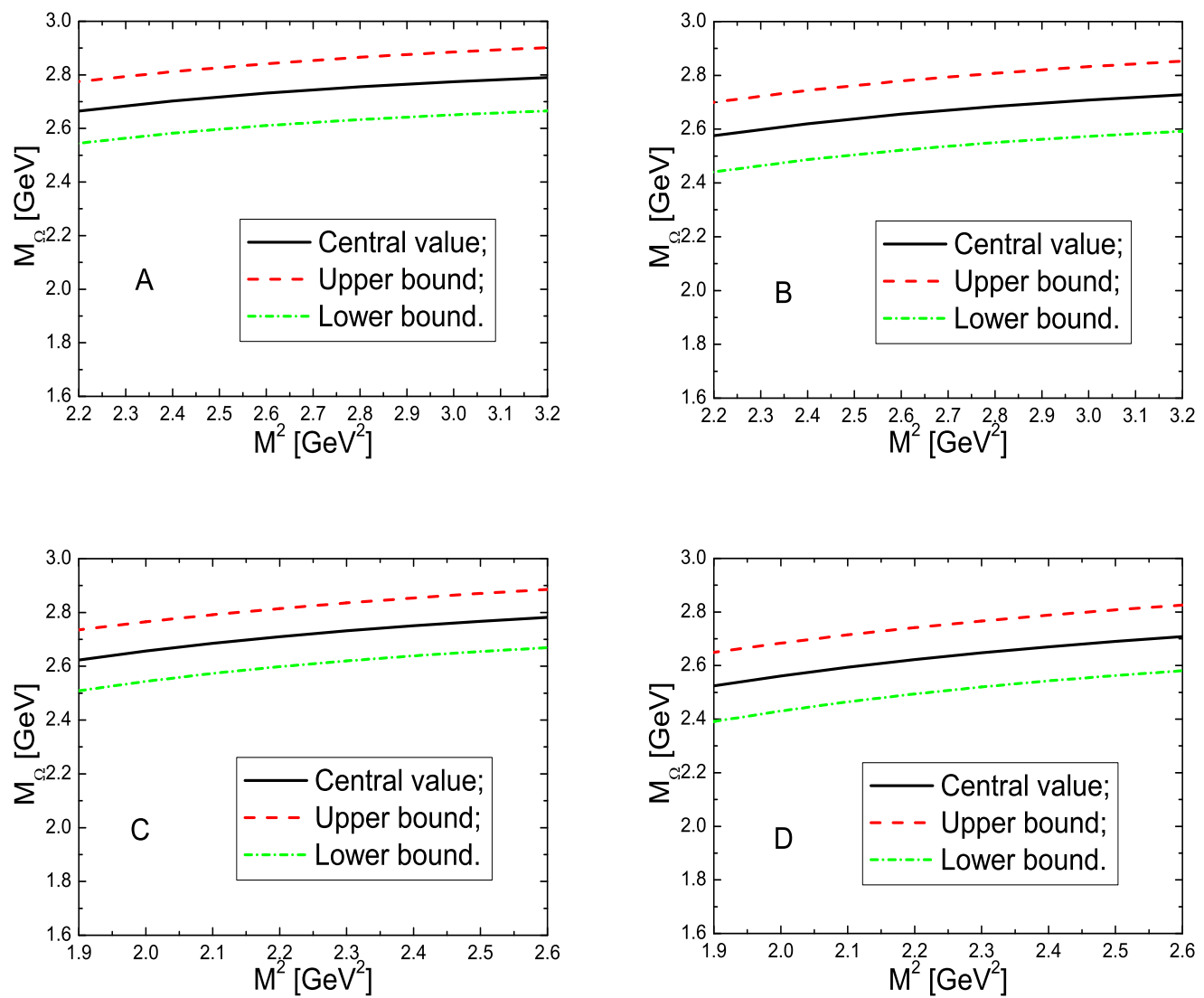

Figure 1: The mass $M_{\Omega_{c}^{0}}$ from the sum rules with different tensor structures and input parameters, $A, B, C$ and $D$ correspond to $\not p \&$ Set I, $1 \&$ Set I, $\not p \&$ Set II and $1 \&$ Set II, respectively.

\begin{tabular}{|c|c|c|c|c|}
\hline \hline & $M_{\Omega_{c}^{0}}(\mathrm{GeV})$ & $M_{\Omega_{b}^{-}}(\mathrm{GeV})$ & $\lambda_{\Omega_{c}^{0}}\left(\mathrm{GeV}^{3}\right)$ & $\lambda_{\Omega_{b}^{-}}\left(\mathrm{GeV}^{3}\right)$ \\
\hline$\not p \&$ Set I & $2.72 \pm 0.18$ & $6.13 \pm 0.12$ & $0.088 \pm 0.026$ & $0.118 \pm 0.026$ \\
\hline 1 \& Set I & $2.66 \pm 0.19$ & $6.09 \pm 0.11$ & $0.064 \pm 0.017$ & $0.088 \pm 0.017$ \\
\hline$\not p \&$ Set II & $2.71 \pm 0.18$ & $6.18 \pm 0.13$ & $0.090 \pm 0.027$ & $0.133 \pm 0.030$ \\
\hline 1 \& Set II & $2.63 \pm 0.20$ & $6.12 \pm 0.13$ & $0.065 \pm 0.017$ & $0.099 \pm 0.019$ \\
\hline$\not$ \& Set III & $2.70 \pm 0.27$ & $6.10 \pm 0.27$ & & \\
\hline 1 \& Set III & $2.50 \pm 0.36$ & $5.90 \pm 0.36$ & & \\
\hline Exp data & $2.6975 \pm 0.0026$ & $6.165 \pm 0.013$ & & \\
\hline \hline
\end{tabular}

Table 4: The masses and pole residues from the sum rules with different tensor structures and input parameters. 

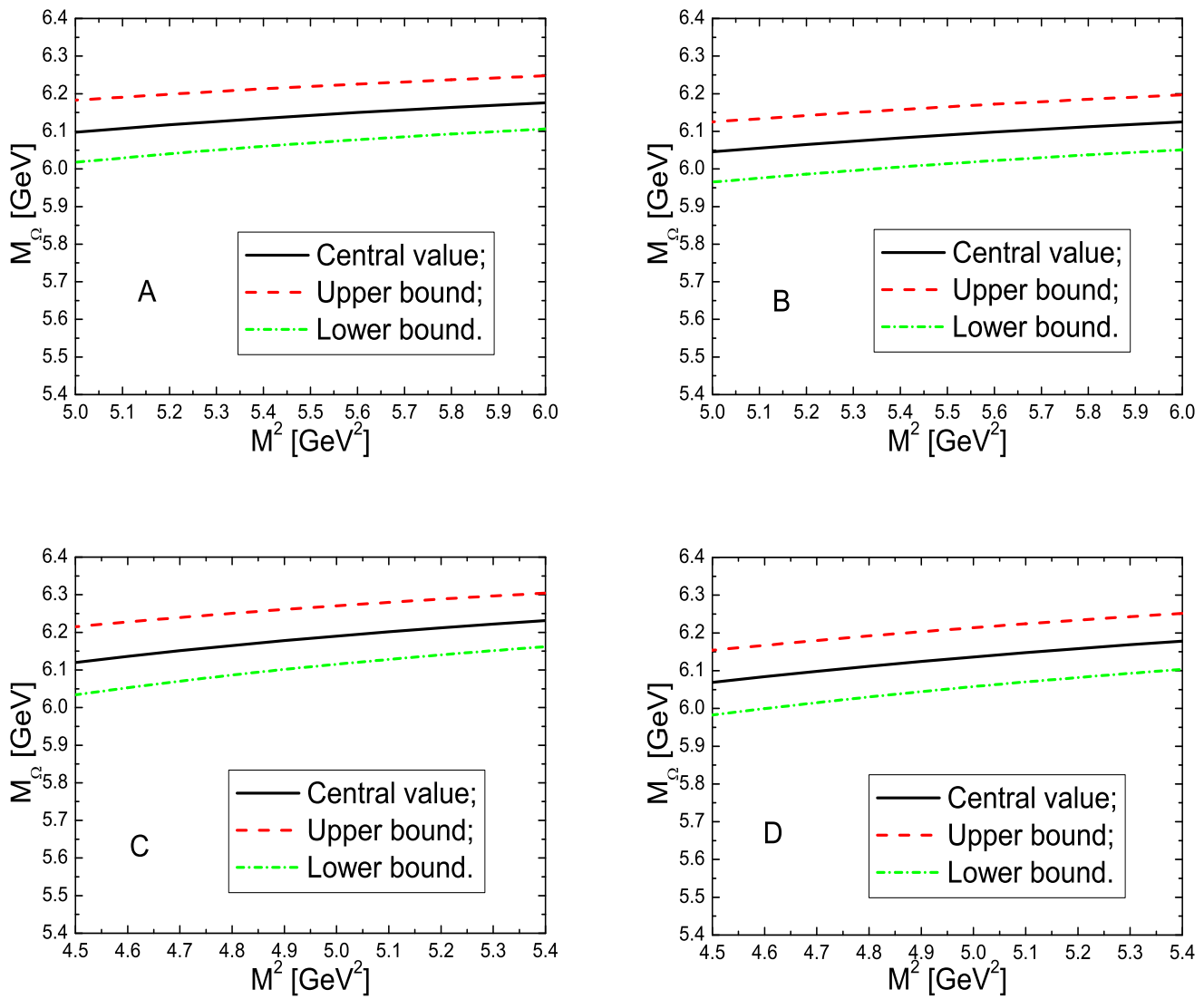

Figure 2: The mass $M_{\Omega_{b}^{-}}$from the sum rules with different tensor structures and input parameters, $A, B, C$ and $D$ correspond to $\not p \&$ Set I, $1 \&$ Set I, $\not p \&$ Set II and $1 \&$ Set II, respectively. 

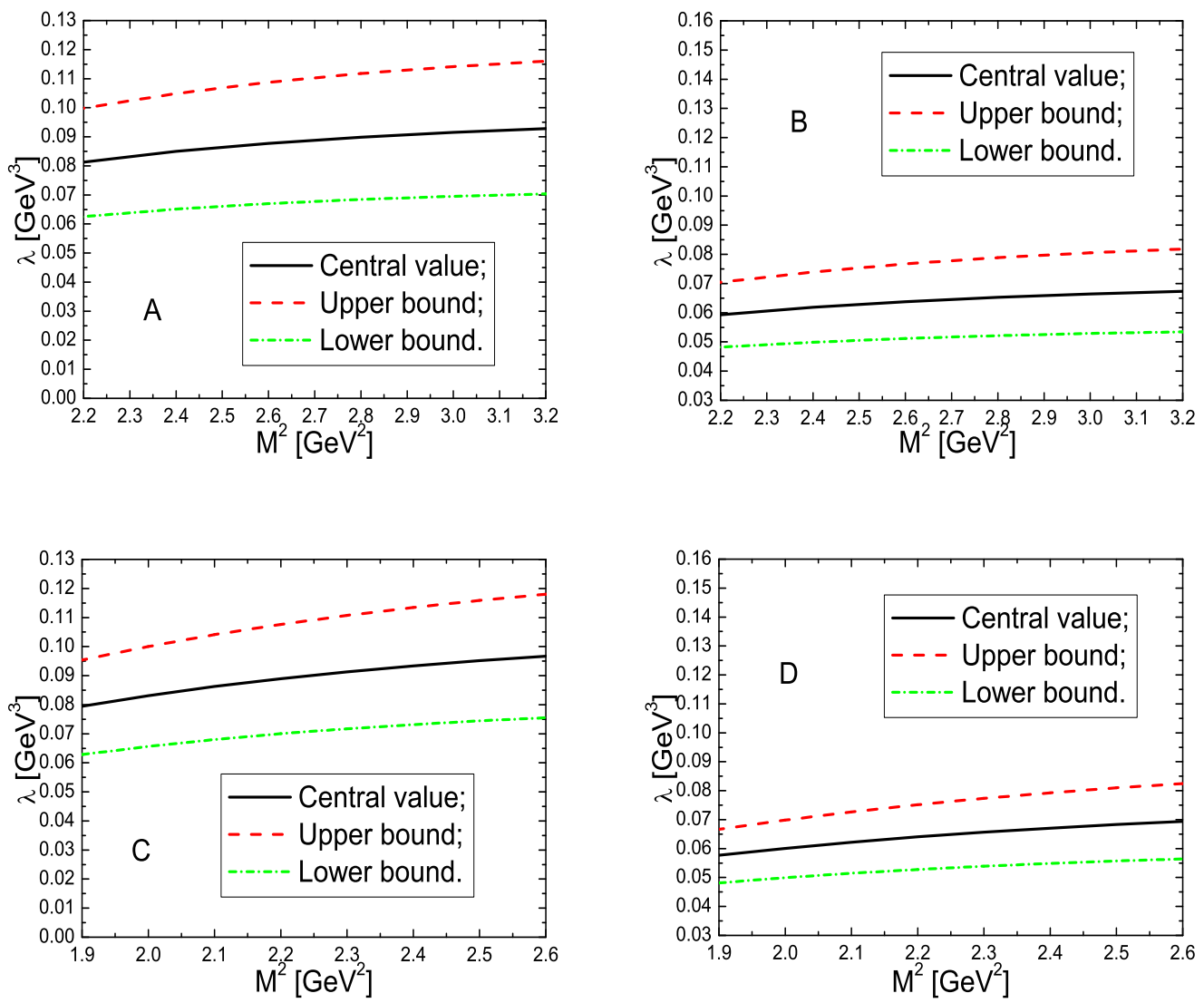

Figure 3: The pole residue $\lambda_{\Omega_{c}}$ from the sum rules with different tensor structures and input parameters, $A, B, C$ and $D$ correspond to $\not p \&$ Set I, $1 \&$ Set I, $\not p \&$ Set II and $1 \&$ Set II, respectively. 

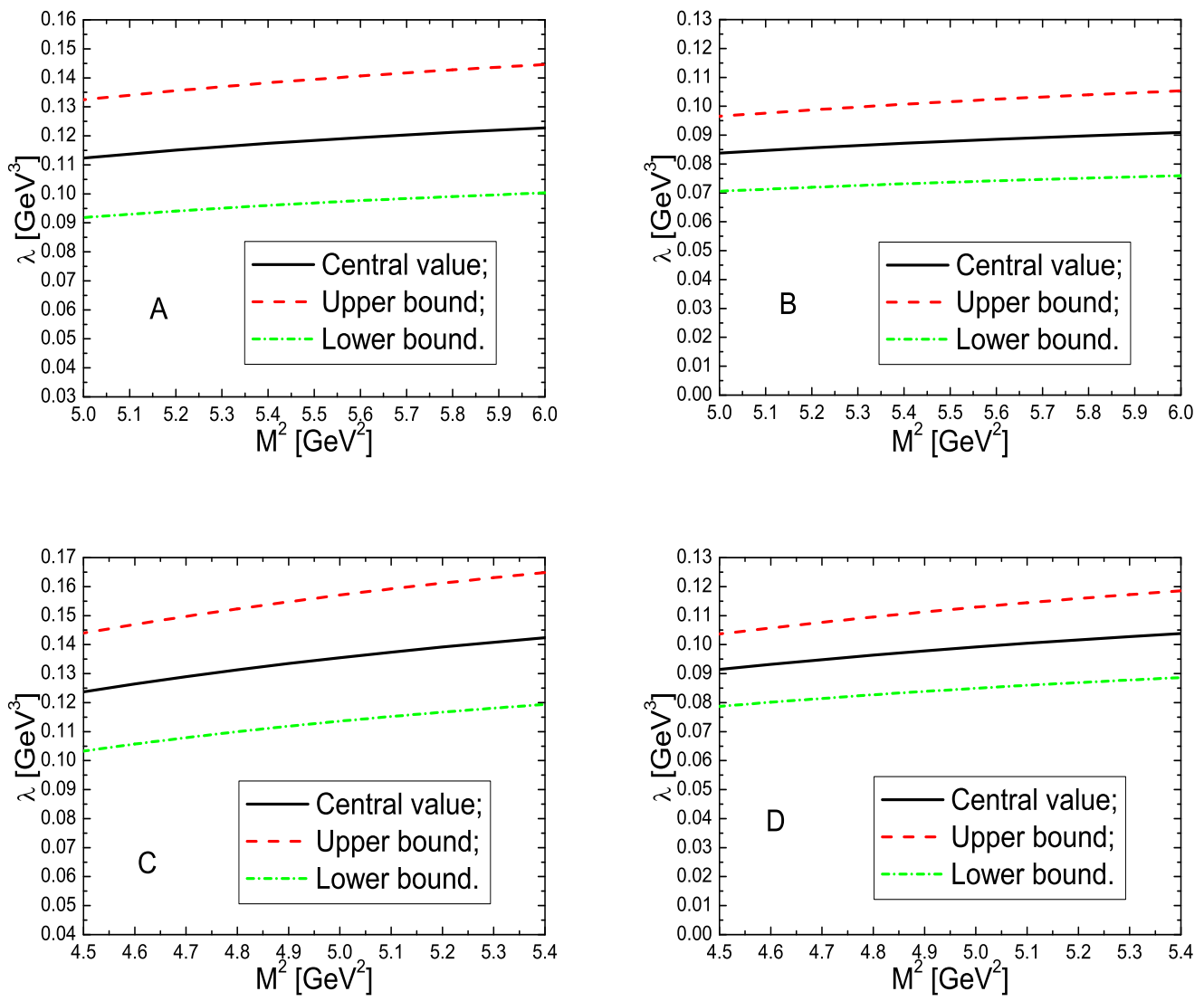

Figure 4: The pole residue $\lambda_{\Omega_{b}}$ from the sum rules with different tensor structures and input parameters, $A, B, C$ and $D$ correspond to $\not p \&$ Set I, $1 \&$ Set I, $\not p \&$ Set II and $1 \&$ Set II, respectively.
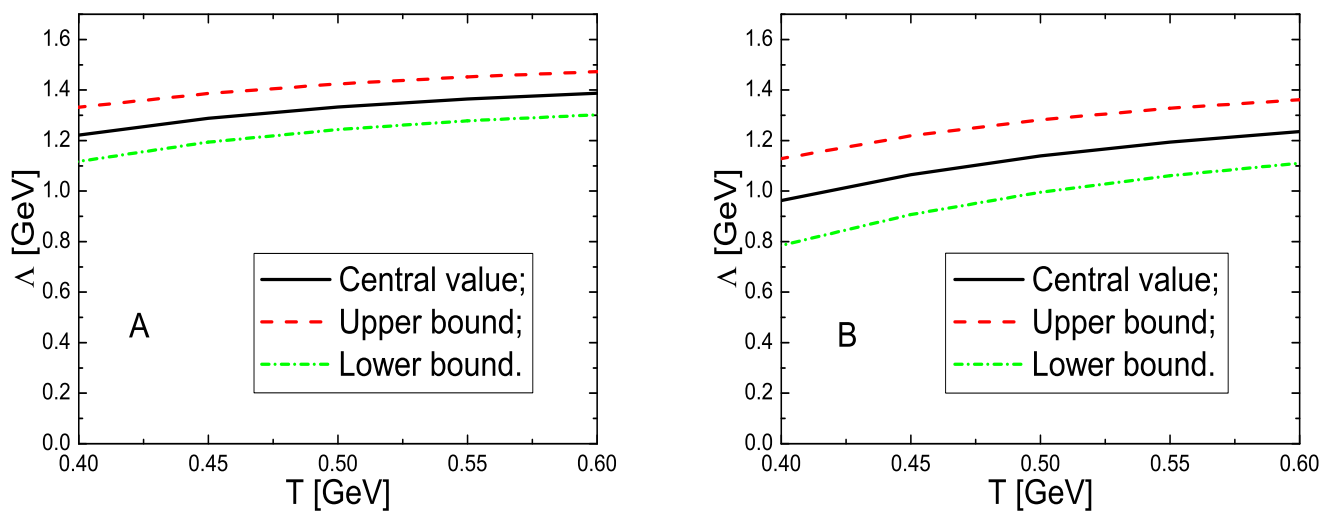

Figure 5: The binding energy $\bar{\Lambda}$ with variation of the Borel parameter $T, A$ and $B$ correspond to the tensor structures $\not 6$ and 1 , respectively. 
data $M_{\Omega_{c}^{0}}=(2.6975 \pm 0.0026) \mathrm{GeV}$ [17], other theoretical predictions also indicate the value is about $2.7 \mathrm{GeV}$ [4, 6, 7, 8, 9, 10, 11, 12, 13, 14. The experimental value $M_{\Omega_{b}^{-}}=6.165 \pm 0.010 \pm 0.013 \mathrm{GeV}$ is about $0.1 \mathrm{GeV}$ larger than the existing theoretical calculations [4, 6, 7, 8, 9, 10, 11, 12, 13, 14] (including the QCD sum rules in the heavy quark effective theory with $1 / m_{Q}$ corrections [13]), our predictions $M_{\Omega_{b}^{-}}=(6.13 \pm 0.12) \mathrm{GeV}$ and $M_{\Omega_{b}^{-}}=(6.18 \pm 0.13) \mathrm{GeV}$ based on the sum rules with the tensor structure $\not p$ are excellent.

The parameters "Set I" satisfy the pole dominance criterion marginally, the parameters "Set II" have larger threshold parameters than that of the "Set I", which may take into account some contributions from the high resonances. More experimental data are needed to select the ideal sum rules. Once reasonable values of the pole residues $\lambda_{\Omega_{c}}$ and $\lambda_{\Omega_{b}}$ are obtained, we can take them as basic input parameters and study the hadronic processes with the (light-cone) QCD sum rules.

In Ref.[20], the tensor structure $\not p$ is chosen, and the spectral density (including the perturbative term, quark condensate term $\langle\bar{s} s\rangle$, mixed condensate term $\left\langle\bar{s} g_{s} \sigma G s\right\rangle$ and gluon condensate term $\left\langle\frac{\alpha_{s} G G}{\pi}\right\rangle$ ) differs from mine, the most evident difference is that the terms of the vacuum condensate $\langle\bar{s} s\rangle$ present in Eq.(8) are absent. I check all calculations carefully and confirm my results. For example, although the term $\langle\bar{s} s\rangle$ vanishes in the heavy quark limit (see Eq.(11)), there indeed exist such a term in the full QCD. The threshold parameter $s_{\Omega_{c}}^{0}=(10.0-11.5) \mathrm{GeV}^{2}$ and the prediction $M_{\Omega_{c}^{0}}=(2.65 \pm 0.25) \mathrm{GeV}$ are consistent with mine, while the lower bound of the threshold parameter $s_{\Omega_{b}}^{0}=(41.0-45.0) \mathrm{GeV}^{2}$ is much smaller than mine, and the prediction $M_{\Omega_{b}^{-}}=(5.82 \pm 0.23) \mathrm{GeV}$ differs from the experimental data remarkably.

The sum rules in the heavy quark limit with the odd structure $\not \varnothing$ also result in excellent values $M_{\Omega_{c}^{0}}=(2.70 \pm 0.27) \mathrm{GeV}$ and $M_{\Omega_{b}^{-}}=(6.10 \pm 0.27) \mathrm{GeV}$, although the uncertainties are large; the values from the even structure 1 are not good enough, see Table 4 , the $1 / m_{Q}$ corrections maybe large. In Ref.[13], the threshold parameter is taken as $\omega_{0}=1.55 \mathrm{GeV}$, which differs from mine remarkably. The predictions (the central values are $M_{\Omega_{c}^{0}}=2.62 \mathrm{GeV}$ and $M_{\Omega_{b}^{-}}=5.97 \mathrm{GeV}$ without the $1 / m_{Q}$ corrections) are smaller than mine about $(0.08-0.10) \mathrm{GeV}$. It is not unexpected, different interpolating currents and input parameters can lead to different results.

In this article, we do not take into account the next-to-leading order corrections to the perturbative term, the corrections maybe large. In the massless limit $m_{s}=$ $m_{u}=m_{d}=0$, we can resort to analytical expressions of the correlation functions for the heavy baryon currents with one heavy quark in the finite mass limit [37, 38] or infinite mass limit [39, 40] to make possible improvement for the predicted masses $M_{\Omega_{a}}$, while the next-to-leading order corrections to the perturbative terms for the baryon currents with three massless quarks are calculated in Refs. [41, 42, 43, 44]. The analytical expressions of the perturbative $\alpha_{s}$ corrections presented in Ref. [37] are lengthy enough; while the expressions in the heavy quark limit $m_{Q} \rightarrow \infty$ are simpler and more easy to deal with, however, the interpolating current differs from mine remarkably [39, 40]. The mass of the $s$ quark plays an important role, the 
$\Omega_{b}$ and $\Sigma_{b}$ have equal masses in the limit $m_{s}=0$, from the experimental data $M_{\Sigma_{b}}=(5807.8 \pm 2.7) \mathrm{MeV}$ [17], we can see that the flavor $S U(3)$ breaking effects (about $350 \mathrm{MeV}$ ) are rather large, $m_{s}=m_{u}=m_{d}=0$ maybe a crude approximation. We prefer another work to study those perturbative effects in detail.

\section{Conclusion}

In this article, we employ the QCD sum rules to calculate the masses and the pole residues of the heavy baryons $\Omega_{c}^{0}$ (css) and $\Omega_{b}^{-}$(bss) including the contributions of the vacuum condensates adding up to dimension six in the operator product expansion. The numerical values $M_{\Omega_{c}^{0}}=(2.72 \pm 0.18) \mathrm{GeV}$ (or $\left.M_{\Omega_{c}^{0}}=(2.71 \pm 0.18) \mathrm{GeV}\right)$ and $M_{\Omega_{b}^{-}}=(6.13 \pm 0.12) \mathrm{GeV}$ ( or $\left.M_{\Omega_{b}^{-}}=(6.18 \pm 0.13) \mathrm{GeV}\right)$ are in good agreement with the experimental data, while the existing theoretical predictions for the mass $M_{\Omega_{b}^{-}}$ is about $0.1 \mathrm{GeV}$ lower than the experimental value. We can take the pole residues $\lambda_{\Omega_{c}}$ and $\lambda_{\Omega_{b}}$ as basic parameters and study the hadronic processes, for example, $\Omega_{Q}^{*} \rightarrow \Omega_{Q} \gamma$

\section{Acknowledgements}

This work is supported by National Natural Science Foundation, Grant Number 10775051, and Program for New Century Excellent Talents in University, Grant Number NCET-07-0282.

\section{References}

[1] J. G. Koerner, D. Pirjol and M. Kraemer, Prog. Part. Nucl. Phys. 33 (1994) 787.

[2] F. Hussain, G. Thompson and J. G. Koerner, arXiv:hep-ph/9311309.

[3] S. Capstick and N. Isgur, Phys. Rev. D34, 2809 (1986).

[4] R. Roncaglia, D. B. Lichtenberg, and E. Predazzi, Phys. Rev. D52, 1722 (1995).

[5] B. Silvestre-Brac, Few-Body Systems 20, 1 (1996).

[6] A. Valcarce, H. Garcilazo, J. Vijande, Eur. Phys. J. A37 (2008) 217.

[7] E. Jenkins, Phys. Rev. D54, 4515 (1996).

[8] K. C. Bowler et al., Phys. Rev. D54, 3619 (1996).

[9] N. Mathur, R. Lewis, and R. M. Woloshyn, Phys. Rev. D66, 014502 (2002). 
[10] D. Ebert, R. N. Faustov, and V. O. Galkin, Phys. Rev. D72, 034026 (2005).

[11] D. Ebert, R. N. Faustov, and V. O. Galkin, Phys. Lett. B659, 612 (2008).

[12] M. Karliner, B. Keren-Zura, H. J. Lipkin, and J. L.Rosner, arXiv:0706.2163; arXiv:0708.4027.

[13] X. Liu, H. X. Chen, Y. R. Liu, A. Hosaka, and S. L. Zhu, Phys. Rev. D77, 014031 (2008).

[14] W. Roberts and M. Pervin, Int. J. Mod. Phys. A23 (2008) 2817.

[15] E. Bagan, M. Chabab, H. G. Dosch, and S. Narison, Phys. Lett. B278, 367 (1992).

[16] E. Bagan, M. Chabab, H. G. Dosch, and S. Narison, Phys. Lett. B287, 176 (1992).

[17] C. Amsler et al, Phys. Lett. B667 (2008) 1.

[18] V. Abazov et al, Phys. Rev. Lett. 101 (2008) 232002.

[19] Z. G. Wang, Eur. Phys. J. C54 (2008) 231.

[20] F. O. Duraes and M. Nielsen, Phys. Lett. B658 (2007) 40.

[21] E. V. Shuryak, Nucl. Phys. B198, 83 (1982).

[22] A. G. Grozin and O. I. Yakovlev, Phys. Lett. B285, 254 (1992).

[23] E. Bagan, M. Chabab, H. G. Dosch and S. Narison, Phys. Lett. B301, 243 (1993).

[24] Y. B. Dai, C. S. Huang, C. Liu and C. D. Lu, Phys. Lett. B371, 99 (1996).

[25] Y. B. Dai, C. S. Huang, M. Q. Huang and C. Liu, Phys. Lett. B387, 379 (1996).

[26] D. W. Wang, M. Q. Huang and C. Z. Li, Phys. Rev. D65, 094036 (2002).

[27] S. L. Zhu, Phys. Rev. D61, 114019 (2000).

[28] C. S. Huang, A. L. Zhang and S. L. Zhu, Phys. Lett. B492, 288 (2000).

[29] D. W. Wang and M. Q. Huang, Phys. Rev. D68, 034019 (2003).

[30] M. A. Shifman, A. I. Vainshtein and V. I. Zakharov, Nucl. Phys. B147 (1979) $385,448$.

[31] L. J. Reinders, H. Rubinstein and S. Yazaki, Phys. Rept. 127 (1985) 1. 
[32] B. L. Ioffe, Nucl. Phys. B188 (1981) 317.

[33] B. L. Ioffe and A. V. Smilga, Nucl. Phys. B232 (1984) 109.

[34] V. M. Belyaev and B. L. Ioffe, Sov. Phys. JETP 56 (1982) 493.

[35] V. M. Belyaev and B. L. Ioffe, Sov. Phys. JETP 57 (1983) 716.

[36] B. L. Ioffe, Prog. Part. Nucl. Phys. 56 (2006) 232.

[37] S. Groote, J. G. Korner and A. A. Pivovarov, Eur. Phys. J. C58 (2008) 355.

[38] S. Groote, J. G. Korner and A. A. Pivovarov, Phys. Rev. D61 (2000) 071501.

[39] S. Groote, J. G. Korner and O. I. Yakovlev, Phys. Rev. D56 (1997) 3943.

[40] S. Groote, J. G. Korner and O. I. Yakovlev, Phys. Rev. D55 (1997) 3016.

[41] A. A. Ovchinnikov, A. A. Pivovarov, L. R. Surguladze, Int. J. Mod. Phys. A6 (1991) 2025.

[42] A. A. Pivovarov and L. R. Surguladze, Nucl. Phys. B360 (1991) 97.

[43] A. A. Ovchinnikov, A. A. Pivovarov and L. R. Surguladze, Sov. J. Nucl. Phys. 48 (1988) 358.

[44] A. A. Pivovarov and L. R. Surguladze, Sov. J. Nucl. Phys. 48 (1989) 1117. 